\title{
Guest editorial: Regulatory signaling in normal and abnormal hematopoiesis
}

\author{
Fumio Arai $^{1}$
}

Received: 25 April 2018 / Accepted: 26 April 2018 / Published online: 4 May 2018

(c) The Japanese Society of Hematology 2018

\begin{abstract}
Stem cells are characterized by their unique ability to both self-renew and differentiate along multiple cellular lineages. Self-renewal and differentiation must be tightly controlled to ensure an appropriate stem cell pool in tissue over the lifetime of an organism. Elucidating the mechanisms controlling stem cell fate and maintenance remains a key challenge in stem cell biology. Hematopoietic stem cells (HSCs) are responsible for the lifelong production of multiple blood cell lineages. To remain functional, these cells must interact with a particular microenvironment, known as the stem cell niche. HSC niches provide various factors, including cytokines, extracellular matrices, nutrients, hormones, and metabolites. These niche factors modulate cell-intrinsic molecular regulatory networks in HSCs. Niche signals also play crucial roles in the induction of HSCs from pluripotent stem cells or vascular endothelial cells. The Progress in Hematology review series in the current issue highlights some critical regulators of HSC maintenance and production.
\end{abstract}

Hematopoietic stem cells (HSCs) are characterized by their ability to self-renew and differentiate along multiple blood cell lineages. Their function is regulated by complex signaling involving various intracellular factors. However, HSC cannot maintain their integrity without stimuli from specific microenvironments called stem cell niches. The HSC niche provides cytokines, extracellular matrices, nutrients, hormones, and metabolites. These extracellular factors modulate cell-intrinsic molecular regulatory networks in HSCs. The HSC niche interaction is thus critical for the lifelong maintenance of blood production. Advances in the study of HSC regulation and maintenance have revealed a number of critical features of these stem cells. In steady-state conditions, HSCs reside in the bone marrow niche and maintain cell cycle quiescence (or dormancy) that keeps the stem cell pool as a reserve against various stresses. Quiescent HSCs are thought to be maintained by low metabolic activity. Energy metabolism in quiescent HSCs largely depends on glycolysis, while activated HSCs synthesize ATP mainly

Fumio Arai

farai@scr.med.kyushu-u.ac.jp

1 Department of Stem Cell Biology and Medicine, Graduate School of Medical Sciences, Kyushu University, 3-1-1 Maidashi, Higashi-ku, Fukuoka 812-8582, Japan through oxidative phosphorylation [1]. The majority of HSCs reside in a hypoxic microenvironment, which sustains quiescent HSCs with a low level of intracellular reactive oxygen species (ROS) and prevents oxidative stress [2]. Metabolic components in the niche are also involved HSC regulation. Recently, Yamazaki and colleague identified the importance of essential amino acids for the maintenance of HSCs in vitro and in vivo [3]. They found that the maintenance of HSCs within the niche is dependent on valine, which, along with cysteine, is also involved in HSC proliferation ex vivo. In this review series, Wilkinson and Yamazaki summarize the metabolic and dietary regulation of HSCs and describe how nutrition affects HSC activity [4]. They also highlight potential applications of metabolic control in bone marrow transplantation and leukemia therapy.

Recent studies have reported that adult HSCs show low rates of protein synthesis. Adult HSCs also exhibit low capacity for protein folding, which promotes the accumulation of un- or misfolded proteins. Unfolded or misfolded proteins induce the unfolded protein response (UPR), which is related to the endoplasmic reticulum (ER) stress response. The ER stress response plays a crucial role in the prevention of hematological malignancy by eliminating abnormal or damaged cells that have a large amount of unfolded or misfolded proteins. In proliferative conditions, HSCs show elevated ER stress, which causes a reduction in self-renewal 
activity. However, in contrast to adult HSCs, fetal liver (FL) HSCs, which proliferate extensively, exhibit higher rates of protein synthesis, but no increase in ER stress. Miharada and colleague showed that bile acids (BA) function as chemical chaperones to prevent ER stress in FL HSCs [5]. They also found that tauroursodeoxycholic acid (TUDCA) reduces ER stress in HSCs during in vitro culture. In this review series, Sigurdsson and Miharada summarize the role of UPR in the regulation of HSCs [6].

Technological advances in BM imaging made it possible to identify the distribution of HSCs in vivo. Most HSCs are found in the perivascular area in BM and interact with sinusoid vessels and arterioles [7]. With the identification of particular HSC localization sites, vascular niches were identified as the primary niche for HSCs in the BM. The vascular niche contributes both to HSC maintenance and to the development of HSCs during embryogenesis. In the embryo, HSCs emerge from hemogenic endothelial cells in the dorsal aorta, and are then maintained in the vascular niche in hematopoietic tissues in the embryo and adult BM. In the recent years, the developmental programs involved in the generation of HSCs from hemogenic endothelial cells have been applied in the ex vivo production of HSCs. Induction of hemogenic endothelial cells from pluripotent stem cells is a critical process in enabling HSC generation. Transcription factors (TFs) that are expressed in HSCs, but not in hemogenic endothelial cells, were transduced into hemogenic endothelial cells, and such TF (RUNX1, SPI1, and five more TFs)-induced cells were transplanted into mouse BM to obtain functional HSCs [8]. Another group independently reported HSC induction from adult endothelial cells by the transduction of TFs (RUNX1, SPI1, and two more TFs) and coculture with vascular niche cells [9]. In both cases, the vascular niche may provide a platform for the induction of functional HSCs from TF-transduced cells. In this issue, Sugimura reviews the function of the vascular niche in the maintenance and induction of HSCs HSCs [10].

Induction of self-renewal and amplification in vitro has been a challenging goal. Expansion of HSCs requires the stimulation of self-renewal. However, repeated cell divisions induce the accumulation of DNA damage that compromises HSC function [11, 12]. This sensitivity to stress-induced DNA damage is a primary obstacle to establishing robust protocols for the ex vivo expansion of functional HSCs. Telomeres are sensitive to such damage, as they are fragile sites in the genome [13, 14]. HSCs lose telomeric DNA with each cell division, which ultimately limits their replicative potential. These cells thus require a protective mechanism for preventing DNA damage response (DDR) at telomeres. The shelterin complex contains six subunit proteins: TRF1, TRF2, POT1, TIN2, TPP1, and Rap1. Shelterin plays a crucial role in the regulation of telomere length and loop structure, as well as in the protection of telomeres from ataxia telangiectasia-mutated (ATM) and ATM- and RAD3-related (ATR)-dependent DDR signaling pathways [15]. Hosokawa et al. recently identified that Pot1a maintains HSC activity by protecting against DNA damage and preventing the production of ROS [16]. Thanks to these protective functions, treatment with exogenous Pot1a supports HSC selfrenewal and function ex vivo and improves the activity of aged HSCs. In a review article in this issue, Hosokawa summarizes the role of telomere maintenance in both normal hematopoiesis and hematological malignancy [17].

As described above, ER stress is responsible for the reduction of the self-renewal activity of HSCs. Appropriate management of ER stress pathway contributes signicantly to the ex vivo expansion of HSCs. Sigurdsson et al. have reported that treatment with TUDCA maintains functional HSCs in vitro by reducing the ER stress response [5] (also see the review by Sigurdsson and Miharada in this issue [6]).

A more detailed understanding of intra- and extracellular regulators of fate determination and maintenance of HSCs, as outlined in this series of Perspectives in Hematology reviews, may shed light on mechanisms involved in the homeostasis of hematopoiesis.

\section{References}

1. Suda T, Takubo K, Semenza GL. Metabolic regulation of hematopoietic stem cells in the hypoxic niche. Cell Stem Cell. 2011;9:298-310.

2. Jang YY, Sharkis SJ. A low level of reactive oxygen species selects for primitive hematopoietic stem cells that may reside in the low-oxygenic niche. Blood. 2007;110:3056-63.

3. Taya Y, Ota Y, Wilkinson AC, Kanazawa A, Watarai H, Kasai M, Nakauchi H, Yamazaki S. Depleting dietary valine permits nonmyeloablative mouse hematopoietic stem cell transplantation. Science. 2016;354:1152-5.

4. Wilkinson AC, Yamazaki S. The hematopoietic stem cell diet. Int J Hematol. 2018. https://doi.org/10.1007/s12185-018-2451-1.

5. Sigurdsson V, Takei H, Soboleva S, Radulovic V, Galeev R, Siva K, Leeb-Lundberg LM, Iida T, Nittono H, Miharada K. Bile acids protect expanding hematopoietic stem cells from unfolded protein stress in fetal liver. Cell Stem Cell 2016;18:522-32.

6. Sigurdsson V, Miharada K. Regulation of unfolded protein response in hematopoietic stem cells. Int J Hematol. 2018; (in press).

7. Morrison SJ, Scadden DT. The bone marrow niche for haematopoietic stem cells. Nature. 2014;505:327-34.

8. Sugimura R, Jha DK, Han A, Soria-Valles C, da Rocha EL, Lu YF, Goettel JA, Serrao E, Rowe RG, Malleshaiah M, Wong I, Sousa P, Zhu TN, Ditadi A, Keller G, Engelman AN, Snapper SB, Doulatov S, Daley GQ. Haematopoietic stem and progenitor cells from human pluripotent stem cells. Nature. 2017;545:432-8.

9. Lis R, Karrasch CC, Poulos MG, Kunar B, Redmond D, Duran JGB, Badwe CR, Schachterle W, Ginsberg M, Xiang J, Tabrizi AR, Shido K, Rosenwaks Z, Elemento O, Speck NA, Butler JM, Scandura JM, Rafii S. Conversion of adult endothelium to immunocompetent haematopoietic stem cells. Nature. 2017;545:439-45. 
10. Sugimura R. The significance and application of vascular niche in the development and maintenance of hematopoietic stem cells. Int J Hematol. 2018. https://doi.org/10.1007/s12185-018-2450-2.

11. Nijnik A, Woodbine L, Marchetti C, Dawson S, Lambe T, Liu C, Rodrigues NP, Crockford TL, Cabuy E, Vindigni A, Enver T, Bell JI, Slijepcevic P, Goodnow CC, Jeggo PA, Cornall RJ. DNA repair is limiting for haematopoietic stem cells during ageing. Nature. 2007;447:686-90.

12. Rossi DJ, Bryder D, Seita J, Nussenzweig A, Hoeijmakers J, Weissman IL. Deficiencies in DNA damage repair limit the function of haematopoietic stem cells with age. Nature. 2007;447:725-29.

13. de Lange T. How telomeres solve the end-protection problem. Science. 2009;326:948-52.
14. Hewitt G, Jurk D, Marques FD, Correia-Melo C, Hardy T, Gackowska A, Anderson R, Taschuk M, Mann J, Passos JF. Telomeres are favoured targets of a persistent DNA damage response in ageing and stress-induced senescence. Nat Commun. 2012;3:708.

15. de Lange T. Shelterin: the protein complex that shapes and safeguards human telomeres. Genes Dev. 2005;19(18):2100-10.

16. Hosokawa K, MacArthur BD, Ikushima YM, Toyama H, Masuhiro Y, Hanazawa S, Suda T, Arai F. The telomere binding protein Pot1 maintains haematopoietic stem cell activity with age. Nat Commun. 2017;8(1):804.

17. Hosokawa $\mathrm{K}$, Arai $\mathrm{F}$. The role of telomere binding molecules for normal and abnormal hematopoiesis. Int J Hematol. 2018. https ://doi.org/10.1007/s12185-018-2432-4. 